\title{
Medicaid expansion and treatment for opioid use disorders in Oregon: an interrupted time-series analysis
}

\author{
Dennis McCarty ${ }^{*}$, Yifan Gu², John W. Mcllveen ${ }^{3}$ and Bonnie K. Lind ${ }^{2}$
}

\begin{abstract}
Background: The study examined the association of the Affordable Care Act's 2014 Medicaid expansion on the use of psychosocial services and pharmacotherapies for opioid use disorders among Oregon Medicaid recipients.

Methods: Logistic regression analysis examined utilization of care before (January 1, 2010-December 31, 2013) and after Medicaid expansion in Oregon (January 1, 2014-December 31, 2016).

Results: Adult membership in the Oregon Health Plan (Medicaid) increased 180\% following Medicaid expansion $(2013=172,539 ; 2014=482,081)$ and the number with a diagnosis of OUD nearly doubled $(2013=6808$; $2014=13,418)$. More individuals received psychosocial services $(2013=4714 ; 2014=8781)$ and medications $(2013=3464 ; 2014=6093)$ for opioid use disorder. The percent of patients receiving psychosocial services (69\% to $65 \%$ ) and the percent of individuals receiving pharmacotherapy (57\% to $45 \%$ ) declined primarily because of a decline in the proportion receiving care in an opioid treatment program $(2013=41 \% ; 2014=33 \%)$. Odds of accessing any psychosocial service increased by $8 \%$ per year from 2010 to 2013 (AOR $=1.08 ; 95 \% \mathrm{Cl} 1.06-1.11$ ) with an 18\% immediate decline associated with Medicaid expansion in 2014 (AOR $=0.82 ; 95 \% \mathrm{Cl} 0.76-0.87$ ). Following Medicaid expansion, the odds of accessing psychosocial services increased 8\% per year (2014 through 2016) (AOR $=1.08 ; 95 \%$ $\mathrm{Cl}$ 1.06-1.11). Use of medications for opioid use disorder found no change in the odds of use in the years prior to Medicaid expansion, an immediate $36 \%(\mathrm{AOR}=0.64 ; 95 \% \mathrm{Cl} 0.60-0.68)$ decline in 2014 , and a $13 \%$ increase per year in 2015 and $2016(\mathrm{AOR}=1.13 ; 95 \% \mathrm{Cl} 1.09-1.16)$.

Conclusion: The number of Medicaid recipients with an opioid use disorder who received psychosocial and pharmacological services increased substantially following Oregon's Medicaid expansion in 2014. There was a decline, however, in the proportion of individuals with an opioid use disorder receiving care in opioid treatment programs.

Keywords: Opioid use disorder, Opioid agonist therapy, Opioid antagonist therapy, Medicaid expansion, Psychosocial services for opioid use disorder, Medication for opioid use disorder
\end{abstract}

\section{Background}

The Affordable Care Act and its expansion of Medicaid eligibility promoted increased coverage for health care and improved access to treatment for alcohol, opioid, and other drug use disorders. Analyses of responses to the National Survey on Drug Use and Health reported reductions in people without insurance but no increase

\footnotetext{
*Correspondence: mccartyd@ohsu.edu

1 OHSU/PSU School of Public Health, Oregon Health \& Science University,

3181 SW Sam Jackson Park Road, Portland, OR 97239, USA

Full list of author information is available at the end of the article
}

in the proportion who entered treatment for substance use disorders [1] and specifically for opioid use disorders [2]. An analysis of Medicaid Drug Utilization files for the years 2011 through 2014, however, observed quarterly increases in prescriptions for buprenorphine and Medicaid spending on buprenorphine; the increase overtime was substantially greater in expansion states [3]. A detailed look at which services individuals receive may shed light on how Medicaid expansion influenced access to care for opioid use disorders. 
The 2014 Medicaid expansion reduced the uninsured rate in Oregon and facilitated potential access to treatment for people with opioid use disorders. In 2014, Oregon had elevated rates of (a) prescribing opioid analgesics (262 opioid prescription fills per 1000 residents), (b) opioid overdose hospitalizations (20.8 overdose hospitalizations per 1000 residents) and 7.1 overdose deaths per 100,000 residents and (c) overdose deaths (7.1 deaths per 1000 residents) (rates obtained from the Oregon Opioid Dashboard) [4]. Fentanyl first appeared in overdose deaths in 2014 with one death and climbed incrementally in 2015 (4 deaths), 2016 (12 deaths) and 2017 (49 deaths) [4]. An analysis of Oregon's Medicaid data examined utilization of psychosocial services for opioid use disorders and medications for opioid use disorders. Analyses assessed change overtime in access to treatments for opioid use disorders on both the number in care and the relative distribution of where care occurred.

\section{Methods}

\section{Data sources}

De-identified Medicaid enrollment, claims and encounter data covering 4 years prior to Medicaid expansion (January 1, 2010 to December 31, 3013) and 3 years post Medicaid expansion (January 1, 2014 to December 31, 2016) were obtained from the Oregon Health Authority under a data use agreement. The Oregon Health and Science University's Institutional Review Board reviewed the study protocol and approved study methods and human subject protections.

\section{Study population}

The analysis was limited to adults with opioid use disorder (identified using ICD-9 and ICD-10 diagnostic codes) who were 18 to 64 years of age and continuously enrolled for at least 11 months in each study year. Individuals who were enrolled in both Medicare and Medicaid were excluded from the analysis because the Medicare data were not included in the data set. Oregon's Medicaid program relies on 15 regional Medicaid managed care plans for $95 \%$ of its beneficiaries. Individuals not enrolled in a regional Medicaid managed care plan were excluded from the analysis.

\section{Outcome measures}

Study outcomes assessed the use of psychosocial services for opioid use disorder (i.e., opioid treatment programs, counseling in specialty outpatient addiction treatment centers, residential and detoxification care in specialty addiction treatment centers and counseling in primary care settings), and the use of medications for opioid use disorder (i.e., buprenorphine, methadone, naltrexone and extended-release naltrexone). Counts of patients receiving one or more of the psychosocial services and medications for opioid use disorder were summed to assess total access to "any psychological services" and "any pharmacotherapy."

ICD-9 and ICD-10 codes identified members with a diagnosed opioid use disorder (OUD). See Additional file 1: Table S1 for specific codes. Procedure codes (Current Procedural Terminology [CPT] Codes and Healthcare Common Procedural Code System), revenue codes, place of service codes, and pharmacy codes specified the use of opioid agonist therapy and other types of treatment received for opioid use disorder. Place of service codes differentiated care provided in primary care settings, outpatient specialty addiction treatment centers, and residential and detoxification services in specialty addiction treatment programs. Only claims that included both a psychosocial service and an OUD diagnosis were included in the count to avoid including psychosocial services received for reasons unrelated to OUD. Pharmacy claims identified individuals with prescriptions for buprenorphine, oral naltrexone and extended-release naltrexone. CPT codes identified individuals receiving extended-release naltrexone (because health plans tend to cover injectable medications as a medical procedure rather than a pharmacy claim) and methadone for opioid use disorder (because methadone cannot be prescribed for use as an opioid agonist therapy).

Federal regulations require opioid treatment programs (treatment centers approved to dispense methadone) to provide psychosocial services as well as opioid agonist therapy. Individuals enrolled in opioid treatment programs, therefore, were included in both the counts of any pharmacotherapy and any psychosocial service. Opioid treatment program services were billed at a bundled rate that covered both medication and psychosocial costs. Specific counseling visits and the type of medication, therefore, were not differentiated in the dataset. Most individuals receiving care in opioid treatment programs receive methadone and all were counted as receiving methadone. See Additional file 1: Table S1 for specific codes.

\section{Independent variables and covariates}

Analytic models included adjustment for study year (coded 1 to 7), post-expansion (coded 0 in years 2010 to 2013 and 1 in years 2014 to 2016), and number of years post expansion (coded 0 in years 2010 to 2013 and sequentially 1 to 3 for years 2014 to 2016). Five covariates assessed associations with patient characteristics: age, gender, race/ethnicity (i.e., African American, American Indian/Alaskan Native, Asian/Pacific Islander, Hispanic/ Latino, White, and unknown), residency (urban versus 
rural), and the presence of co-occurring psychiatric diagnoses (yes versus no).

\section{Analysis}

Data were aggregated by calendar year. The unit of analysis was the person year. Logistic regression analyses assessed the association between Medicaid expansion, patient characteristics and the receipt of psychosocial services and medications for opioid use disorder. To access the impact of Medicaid expansion, the interrupted time series analysis [5] included the three measures of time: (1) study year (to control for secular trends), (2) Medicaid expansion (to test for immediate effects of expansion) and (3) years post Medicaid expansion (to test for continuing change and change in slope post expansion). A sensitivity analysis removed opioid treatment programs from the count of any psychosocial services to understand how inclusion of opioid treatment programs affected the proportion of individuals who received psychosocial services. Standard errors were clustered at the individual level to address the correlation between the longitudinal observations for members with multiple observations. Data management and analysis used $\mathrm{R}$ version 3.5.1 software.

\section{Results}

Numbers diagnosed with opioid use disorder

Medicaid expansion was associated with a 1.8-fold increase in adults enrolled in Oregon Medicaid comparing $2013(n=172,539)$ to $2014(n=482,081)$. The number of individuals with a diagnostic indicator of an opioid use disorder nearly doubled $(2013=6808 ; 2014=13,418)$ following Medicaid expansion and increased to more than 15,000 in $2015(n=15,251)$ and $2016(n=15,021)$. Table 1 summarizes the numbers and characteristics of the Medicaid enrollees with a diagnosis of opioid use disorder by year (2010-2016).

\section{Counts of psychosocial services}

The number of Medicaid recipients receiving any psychosocial service for opioid use disorder increased $86 \%$ following Medicaid expansion $(2013=4714 ; 2014=8781)$ and increased to more than 10,000 in $2015(n=10,028)$ and $2016(n=10,193)$. Increased access was observed in all psychosocial service settings between 2013 and 2014: specialty outpatient ( $n=2106$ vs. $n=3974)$, specialty residential $(n=675$ vs. $n=1787)$, primary care $(n=125$ vs. $n=478)$, and care in opioid treatment programs $(n=2786$ vs. $n=4394$ ). Table 2 details the annual counts and Fig. 1 plots the change over time.

Table 1 Characteristics of Medicaid recipients with an OUD diagnosis

\begin{tabular}{|c|c|c|c|c|c|c|c|c|c|c|c|c|c|c|}
\hline & \multicolumn{2}{|l|}{2010} & \multicolumn{2}{|l|}{2011} & \multicolumn{2}{|l|}{2012} & \multicolumn{2}{|l|}{2013} & \multicolumn{2}{|l|}{2014} & \multicolumn{2}{|l|}{2015} & \multicolumn{2}{|l|}{2016} \\
\hline & $N$ & $\%$ & $\mathrm{~N}$ & $\%$ & $N$ & $\%$ & $\mathrm{~N}$ & $\%$ & $N$ & $\%$ & $\mathrm{~N}$ & $\%$ & $\mathbf{N}$ & $\%$ \\
\hline OUD population & 3653 & 100 & 5733 & 100 & 6235 & 100 & 6808 & 100 & 13,418 & 100 & 15,251 & 100 & 15,021 & 100 \\
\hline Age (mean) & 40.8 & $\mathrm{n} / \mathrm{a}$ & 39.5 & $\mathrm{n} / \mathrm{a}$ & 39.2 & $\mathrm{n} / \mathrm{a}$ & 39.3 & $\mathrm{n} / \mathrm{a}$ & 37.9 & n/a & 38.5 & $\mathrm{n} / \mathrm{a}$ & 38.9 & $\mathrm{n} / \mathrm{a}$ \\
\hline $18-24$ years & 332 & 9.1 & 598 & 10.4 & 652 & 10.5 & 724 & 10.6 & 1556 & 11.6 & 1501 & 9.8 & 1248 & 8.3 \\
\hline $25-34$ years & 1007 & 27.6 & 1819 & 31.7 & 2049 & 32.9 & 2164 & 31.8 & 4864 & 36.2 & 5439 & 35.7 & 5405 & 36.0 \\
\hline $35-44$ years & 792 & 21.7 & 1239 & 21.6 & 1350 & 21.7 & 1575 & 23.1 & 3006 & 22.4 & 3520 & 23.1 & 3618 & 24.1 \\
\hline $45-54$ years & 891 & 24.4 & 1246 & 21.7 & 1291 & 20.7 & 1299 & 19.1 & 2315 & 17.3 & 2670 & 17.5 & 2538 & 16.9 \\
\hline 55-64 years & 631 & 17.3 & 831 & 14.5 & 893 & 14.3 & 1046 & 15.4 & 1677 & 12.5 & 2121 & 13.9 & 2212 & 14.7 \\
\hline \multicolumn{15}{|l|}{ Gender } \\
\hline Male & 1173 & 32.1 & 2085 & 36.4 & 2245 & 36.0 & 2389 & 35.1 & 6245 & 46.5 & 7144 & 46.8 & 6860 & 45.7 \\
\hline \multicolumn{15}{|l|}{ Race/ethnicity } \\
\hline White & 2994 & 82.0 & 4724 & 82.4 & 5175 & 83.0 & 5658 & 83.1 & 10,670 & 79.5 & 12,262 & 80.4 & 12,021 & 80.0 \\
\hline Hispanic & 173 & 4.7 & 292 & 5.1 & 286 & 4.6 & 338 & 5.0 & 1090 & 8.1 & 1158 & 7.6 & 1081 & 7.2 \\
\hline African American & 232 & 6.4 & 302 & 5.3 & 309 & 5.0 & 340 & 5.0 & 495 & 3.7 & 551 & 3.6 & 552 & 3.7 \\
\hline American Indian/Alaskan Native & 130 & 3.6 & 192 & 3.3 & 218 & 3.5 & 238 & 3.5 & 477 & 3.6 & 506 & 3.3 & 520 & 3.5 \\
\hline Asian/Pacific Islander & 17 & 0.5 & 40 & 0.7 & 54 & 0.9 & 48 & 0.7 & 125 & 0.9 & 146 & 1.0 & 136 & 0.9 \\
\hline Other/unknown & 107 & 2.9 & 183 & 3.2 & 193 & 3.1 & 186 & 2.7 & 561 & 4.2 & 628 & 4.1 & 711 & 4.7 \\
\hline \multicolumn{15}{|l|}{ Geography } \\
\hline Rural & 968 & 26.5 & 1505 & 26.3 & 1691 & 27.1 & 1957 & 28.7 & 3875 & 28.9 & 4591 & 30.1 & 4661 & 31.0 \\
\hline Psychiatric disorder & 2545 & 69.7 & 3926 & 68.5 & 4156 & 66.7 & 4452 & 65.4 & 7536 & 56.2 & 8658 & 56.8 & 8723 & 58.1 \\
\hline
\end{tabular}

Assessed among continuously enrolled adults (18-64) with an OUD diagnosis, not dually eligible for Medicaid and Medicare, and enrolled in a regional Medicaid health plan 
Table 2 Counts and percentages of Medicaid recipients with OUD who received psychosocial services and/ or pharmacotherapy

\begin{tabular}{|c|c|c|c|c|c|c|c|}
\hline & 2010 & 2011 & 2012 & 2013 & 2014 & 2015 & 2016 \\
\hline Total study population (N) & 107,398 & 158,677 & 167,939 & 172,539 & 482,081 & 491,125 & 455,607 \\
\hline \multicolumn{8}{|l|}{ Opioid use disorder (N, \%) } \\
\hline OUD diagnosis & $3653(3.4)$ & $5733(3.6)$ & $6235(3.7)$ & $6808(4.0)$ & $13,418(2.8)$ & $15,251(3.1)$ & $15,021(3.3)$ \\
\hline \multicolumn{8}{|l|}{ Psychosocial services $(\mathrm{N}, \%)$} \\
\hline Residential and detoxification & $83(2.3)$ & $221(3.9)$ & $414(6.6)$ & $675(9.9)$ & $1787(13.3)$ & $1844(12.1)$ & $2078(13.8)$ \\
\hline Opioid treatment program & $1651(45.2)$ & 2555 (44.6) & $2747(44.1)$ & $2786(40.9)$ & $4394(32.8)$ & $4694(30.8)$ & $4683(31.2)$ \\
\hline Primary care & $123(3.4)$ & $81(1.4)$ & $98(1.6)$ & $125(1.8)$ & $478(3.6)$ & $594(3.9)$ & $938(6.2)$ \\
\hline Specialty outpatient & $930(25.5)$ & $1460(25.5)$ & $1620(26.0)$ & $2106(30.9)$ & 3974 (29.6) & $4898(32.1)$ & $4882(32.5)$ \\
\hline Any psychosocial services & $2386(65.3)$ & $3682(64.2)$ & $4106(65.9)$ & $4714(69.2)$ & $8781(65.4)$ & $10,028(65.8)$ & $10,193(67.9)$ \\
\hline \multicolumn{8}{|l|}{ Pharmacotherapy (N, \%) } \\
\hline Buprenorphine & $206(5.6)$ & $396(6.9)$ & $511(8.2)$ & $634(9.3)$ & $1483(11.1)$ & $2006(13.2)$ & $2335(15.5)$ \\
\hline Methadone & $1651(45.2)$ & 2555 (44.6) & $2747(44.1)$ & $2786(40.9)$ & 4394 (32.8) & $4694(30.8)$ & $4683(31.2)$ \\
\hline Oral naltrexone & $4(0.1)$ & $13(0.2)$ & $25(0.4)$ & $38(0.6)$ & $83(0.6)$ & $173(1.1)$ & $304(2.0)$ \\
\hline Naltrexone ext-release & $2(0.1)$ & $1(0.0)$ & $51(0.8)$ & $134(2.0)$ & $459(3.4)$ & $711(4.7)$ & $941(6.3)$ \\
\hline Any pharmacotherapy & $1844(50.5)$ & $2925(51.0)$ & $3270(52.5)$ & $3464(50.9)$ & $6093(45.4)$ & 7075 (46.4) & 7617 (50.7) \\
\hline
\end{tabular}

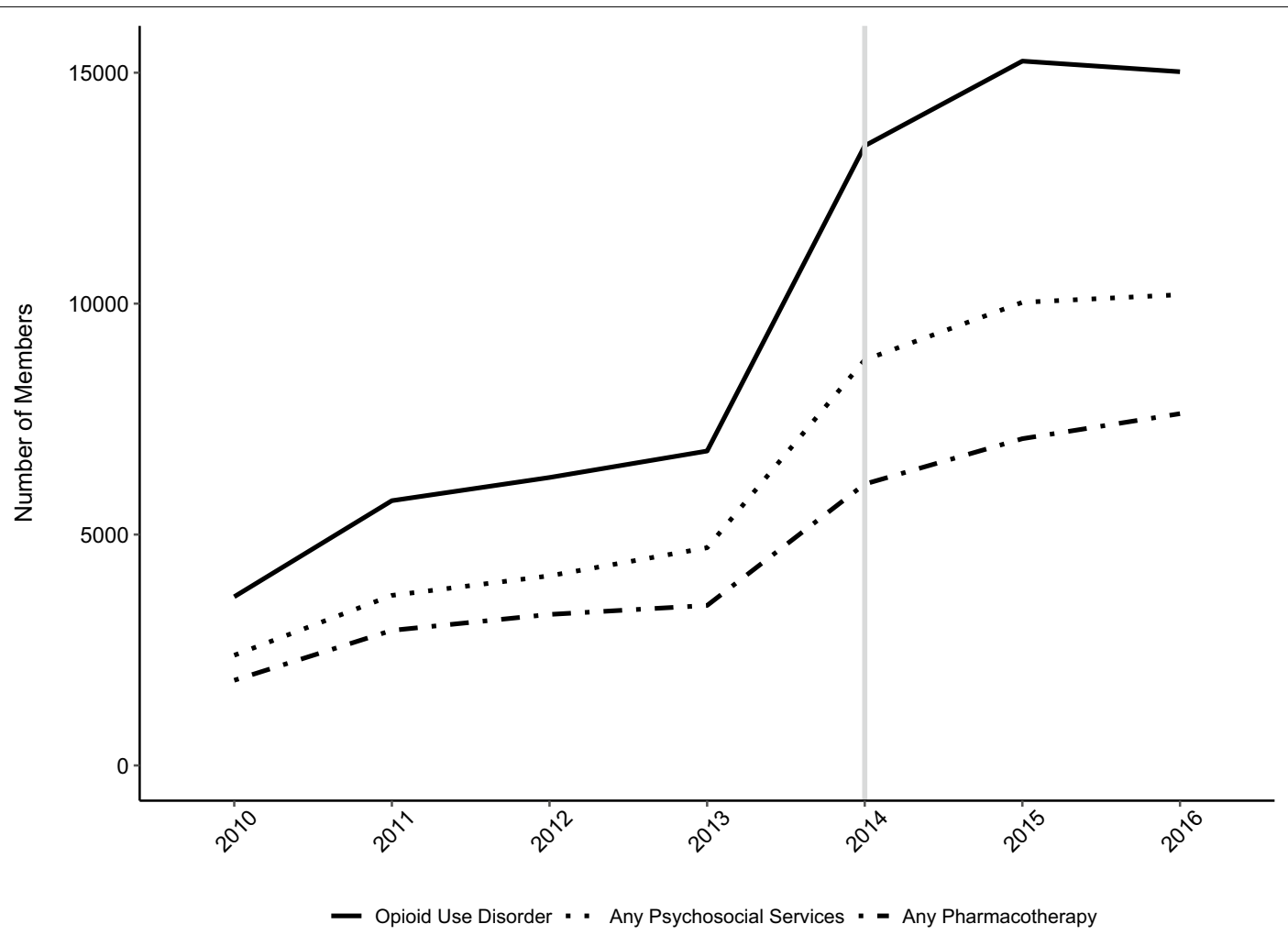

Fig. 1 Change in numbers with an opioid use disorder, receiving psychosocial services and/or medications for opioid use disorders (2010 to 2016)

\section{Counts of pharmacotherapy}

More individuals with opioid use disorder received pharmacotherapy with a medication for opioid use disorder between 2013 and 2014: buprenorphine ( $n=634$ vs. $n=1483$ ), methadone ( $n=2786$ vs. $n=4394)$, extendedrelease naltrexone $(n=134$ vs. $n=459)$ and oral naltrexone $(n=38$ vs. $n=83)$. Access to pharmacotherapy for opioid use disorder improved to more than 7000 
individuals in $2015(n=7075)$ and $2016(n=7617)$. See Table 2 and Fig. 1.

\section{Proportion using psychosocial services}

The proportion of individuals with a diagnostic indication of an opioid use disorder receiving any psychosocial services for opioid use disorder increased to 69\% in 2013 and declined to $65 \%$ in 2014 with subsequent increases to $66 \%$ (2015) and 68\% (2016). The proportion in specialty outpatient care grew from $26 \%(2010-2012)$ to $30 \%$ (2013-2014) to 32\% (2015-2016).

Logistic regression analyses assessed change over the study period and tested the impact of Medicaid expansion. Odds of any psychosocial service use increased $8 \%$ $(\mathrm{AOR}=1.08,95 \%$ CI 1.06-1.11) per year over the study period with an $18 \%$ immediate decline $(A O R=0.82,95 \%$ CI 0.76-0.87) associated with Medicaid expansion and a nonsignificant change to the overall slope in 2015 and 2016. The adjusted odds ratios for the covariates suggested that men were less likely than women to receive psychosocial services, urban residents were more likely to receive care, and people with psychiatric diagnoses were less likely to enter psychosocial services. Table 3 summarizes the logistic regression analyses. The unadjusted trends over time for access to psychosocial services are plotted in Fig. 2. Patterns varied but the general trend was an increase over time with a dip at Medicaid expansion. The 2014 decline into the proportion receiving any psychosocial service appears to be related to the 2013 to 2014 decrease in the relative use of opioid treatment programs. The sensitivity analysis found the use of psychosocial services increased year by year over the study period when services in opioid treatment programs were removed from the analysis. See Additional file 1: Table S2.

\section{Proportion using a medication for opioid use disorder}

Despite the increase in the number of Medicaid recipients receiving opioid agonist or opioid antagonist therapy, the proportion of members with a diagnostic indication of an opioid use disorder using a medication for opioid use disorder declined following Medicaid expansion $(2013=51 \% ; 2014=45 \%)$. See Table 2 .

In the multi-variable logistic regression analysis of use of any pharmacotherapy, odds of use were unchanged in the years prior to expansion $(\mathrm{AOR}=1.01,95 \% \mathrm{CI}$ 0.99-1.03). Medicaid expansion, however, was associated with an immediate $36 \%$ decline in the likelihood of using medication (AOR $=0.64$, 95\% CI 0.60-0.68). Following the immediate decline, the odds of any pharmacotherapy increased by $13 \%$ per year in 2015 and $2016(\mathrm{AOR}=1.13$, 95\% CI 1.09-1.16). In addition, men, American Indians, Asians or Pacific islanders, and Hispanic (compared to White) and individuals with psychiatric disorders were less likely to receive medication while urban residents were more likely to receive a medication for opioid use disorder. See Table 3 for the logistic regression results. Overall, there was a steady decline in the proportion of individuals receiving methadone and an increase in the use of buprenorphine and extended-release naltrexone. Unadjusted trends over time are displayed in Fig. 3. See Supplemental Table 3 for the regression analyses on

Table 3 Multi-variable logistic regression analysis of the use of any psychosocial services or any pharmacotherapy among people with OUD

\begin{tabular}{|c|c|c|c|c|}
\hline & \multicolumn{2}{|c|}{ Any psychosocial services } & \multicolumn{2}{|l|}{ Any pharmacotherapy } \\
\hline & Adjusted- odds ratio & $95 \% \mathrm{Cl}$ & Adjusted-odds ratio & $95 \% \mathrm{Cl}$ \\
\hline Year & 1.08 & $(1.06,1.01)$ & 1.01 & $(0.99,1.03)$ \\
\hline Post expansion (reference pre-expansion) & 0.82 & $(0.76,0.64)$ & 0.64 & $(0.60,0.68)$ \\
\hline Year after expansion & 0.98 & $(0.95,1.13)$ & 1.13 & $(1.09,1.16)$ \\
\hline Age & 1.01 & $(1.01,1.00)$ & 1.00 & $(1.00,1.00)$ \\
\hline Gender: male (reference female) & 0.93 & $(0.89,0.94)$ & 0.94 & $(0.89,0.98)$ \\
\hline Residence: urban (reference rural) & 1.97 & $(1.88,2.57)$ & 2.57 & $(2.44,2.71)$ \\
\hline Race: African American & 0.95 & $(0.84,1.06)$ & 1.01 & $(0.89,1.14)$ \\
\hline Race: American Indian/Alaskan Native & 0.90 & $(0.80,1.01)$ & 0.84 & $(0.73,0.96)$ \\
\hline Race: Asian/Pacific Islander & 0.91 & $(0.72,1.15)$ & 0.94 & $(0.73,1.20)$ \\
\hline Race: hispanic & 0.95 & $(0.87,1.04)$ & 0.96 & $(0.88,1.06)$ \\
\hline Race: other/unknown & 0.87 & $(0.79,0.97)$ & 0.91 & $(0.81,1.03)$ \\
\hline $\begin{array}{l}\text { Race: white (reference) } \\
\text { Psychiatric disorder: yes (reference no) }\end{array}$ & 0.84 & $(0.81,0.87)$ & 0.63 & $(0.60,0.65)$ \\
\hline
\end{tabular}

"Year" is coded 1 to 7; "Post expansion" is coded 0 for years 2010 to 2013, and 1 for years 2014 to 2016; "Year after expansion" is coded 0 for years 2010 to 2013 , and 1 , 2, 3 for years 2014 to 2016; "Age" is in years; the reference level of gender is female; the reference level of "Residence" is rural; the reference level of "Race" is White; the reference level of "Psychiatric Disorder" is "no psychiatric disorder diagnosis" 


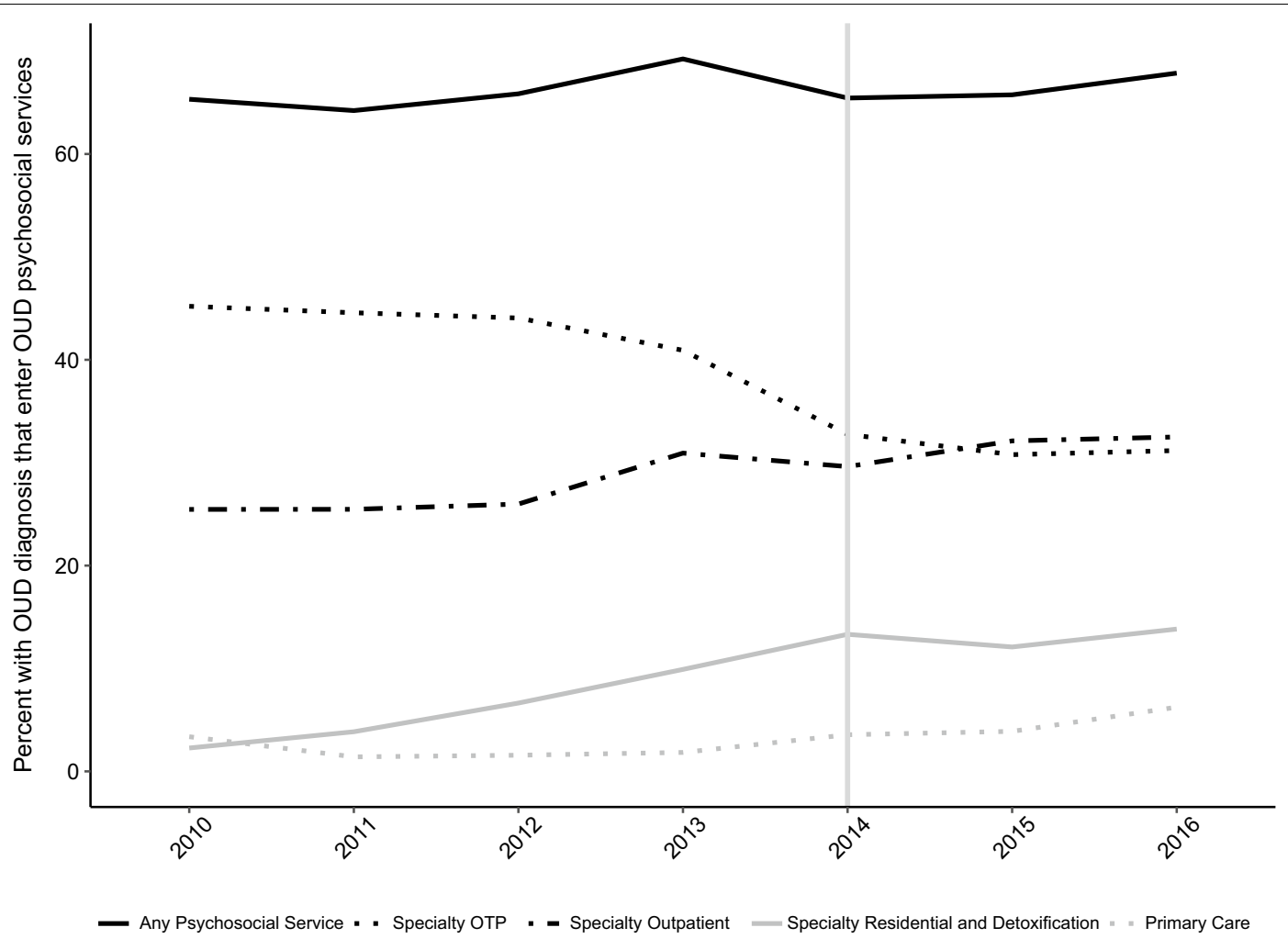

Fig. 2 Change in proportion of individuals with opioid use disorders receiving psychosocial services (2010 to 2016)

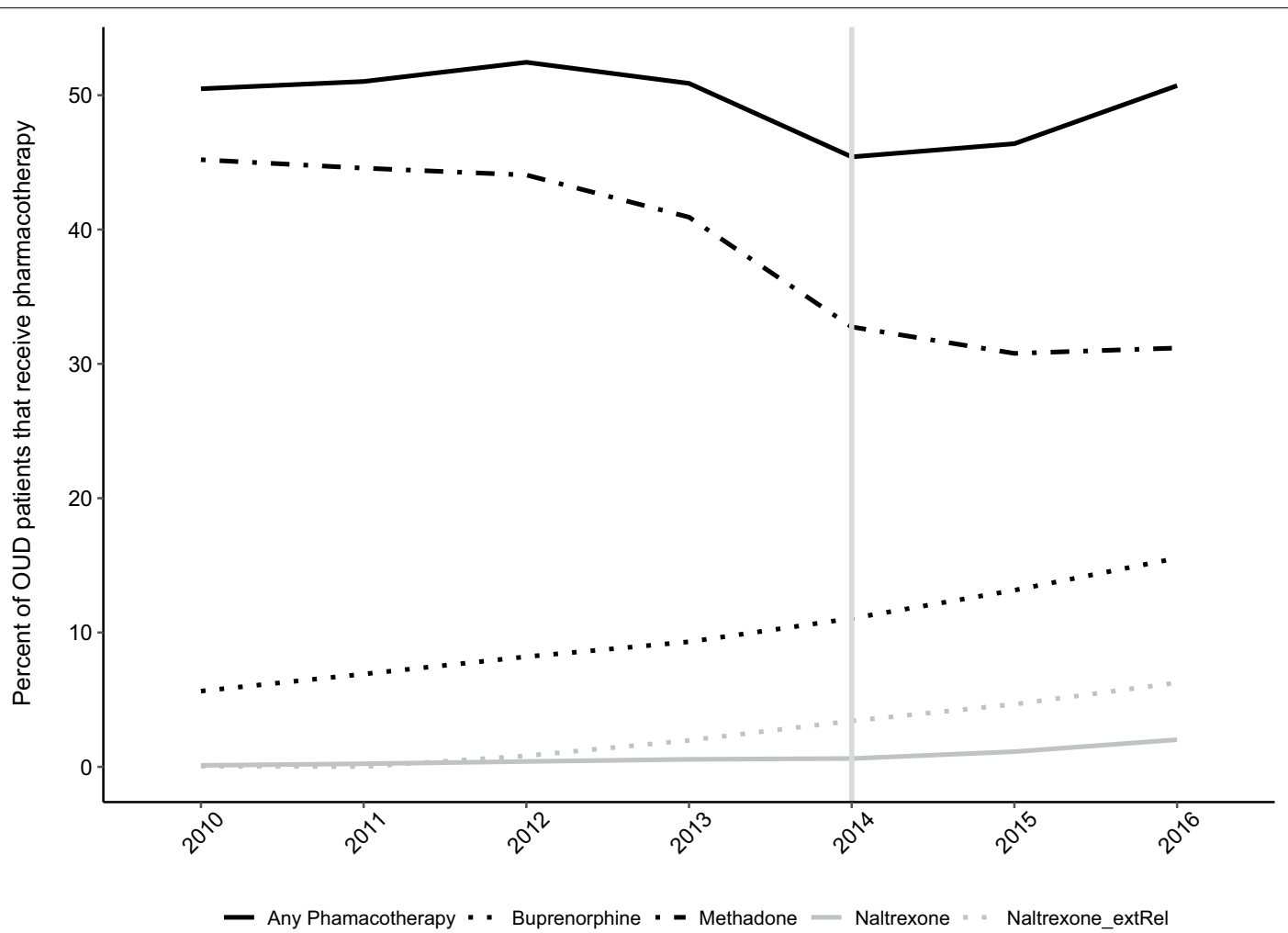

Fig. 3 Change in proportion of individuals with opioid use disorders receiving medications for opioid use disorders (2010 to 2016) 
buprenorphine and methadone. Regression analyses for naltrexone use were not conducted because counts were too small for stable models.

\section{Discussion}

Oregon's Medicaid expansion was associated with a nearly twofold increase in the number of individuals enrolled in Medicaid health plans, the number of individuals with a diagnosis indicating an opioid use disorder, receiving psychosocial services, and receiving an opioid agonist or an opioid antagonist medication to support recovery from opioid use disorder. The overall increase in Medicaid recipients receiving care for opioid use disorder illustrates the value of Medicaid expansion and its role in addressing the opioid epidemic.

The decline in the proportion of Medicaid recipients receiving care in opioid treatment programs from $45 \%$ (2010) to $31 \%$ (2016) reflects a long-term change in the use of methadone. Increased use of buprenorphine $(2010=6 \% ; 2016=16 \%)$ and naltrexone $(2010=0 \%$; $2016=8 \%$ ) offset the decline in the use of methadone and, overall, the rate of individuals using opioid agonist or antagonist medications was stable at about $50 \%$. The relatively abrupt decline in the proportion enrolled in opioid treatment programs between 2013 (41\%) to 2014 (33\%) could reflect greater enrollment in Medicaid from rural communities or increased wait lists within opioid treatment programs. The proportion of Medicaid recipients with an opioid use disorder from rural zip codes, however, was unchanged at 29\% in 2013 and 2014 (see Table 1). The State Opioid Treatment Authority does not maintain a wait list and believes that in most areas of the state admissions to opioid treatment programs occur within days of seeking care. Oregon's Medicaid Managed Care plans require prior authorization for medications for opioid use disorder and some may require individuals to fail first at less expensive therapies; these policies may have contributed to the proportional decline in the use of opioid treatment programs. Another possibility is that the individuals covered under Medicaid expansion may have been new to care and reluctant to initiate care within an opioid treatment program.

Medicaid expansion was also associated with increases in the proportion receiving residential detoxification and post-detoxification care, and a small increase in the proportion receiving counseling services in primary care settings. The distributional shifts may reflect patient preferences, improved choice and the difficulty of matching patients to appropriate levels of care.

Medicaid recipients in rural communities, people with a psychiatric disorder, and men were less likely to access psychosocial and opioid agonist and antagonist medications for opioid use disorder. American Indians were also less likely to access opioid agonist and antagonist medications. Geography and long travel times inhibit access to care in rural communities. Oregon's American Indian tribes have been reluctant to use opioid agonist medication and efforts to facilitate use of medication continue in tribal settings. Historically, Oregon's specialty addiction treatment services have not been licensed to treat mental health disorders and this may account for the reduced access among individuals with psychiatric disorders.

\section{Limitations}

The analysis is limited because the data were from a single state in the northwest region of the United States. Generalizability is uncertain to other states and regions. Substance use disorders are frequently under diagnosed and, especially in 2014, underdiagnoses within the Medicaid expansion population may have distorted the proportions receiving care. The proportional decline in the use of methadone and the increased use of buprenorphine are under-estimated because the data do not break out the use of specific medications in opioid treatment programs. The ICD-9 and ICD-10 diagnostic codes used to identify patients with opioid use disorder assess opioid dependence rather than the current American Psychiatric Association's definition of opioid use disorder [6]. Finally, the study is a retrospective cohort analysis that cannot establish causation.

\section{Conclusion}

Oregon's 2014 Medicaid expansion was associated with increased access to and utilization of psychosocial services for opioid use disorders and use of medications for opioid use disorder. The numbers accessing care doubled and reflected changes in the services provided to treat opioid use disorders.

\section{Additional file}

Additional file 1. Additional tables.

Abbreviations

AOR: adjusted odds ratio; $\mathrm{Cl}$ : confidence interval; $\mathrm{CPT}$ : current procedural terminology; ICD: International Classification of Diseases; OUD: opioid use disorder.

\section{Acknowledgements}

Jiaming (Jerry) Huang helped to complete the final draft of the manuscript. An early version of the analysis was presented at the 2018 Annual Meeting of the College on Problems of Drug Dependence. We also thank the anonymous peer reviewers whose comments and suggestions strengthened the manuscript. 


\section{Authors' contributions}

DM led the conception of the analysis and the preparation of an initial draft of the manuscript. YG completed the statistical analysis under the supervision of BKL. JWM provided data on methadone and buprenorphine providers in Oregon. All authors read and approved the final manuscript.

\section{Funding}

Awards from the National Institutes of Health supported data analysis and preparation of the manuscript (R33 DA035640, UG1 DA015815).

\section{Availability of data and materials}

A data use agreement with the Oregon Health Authority authorized the use of the data for this study and does not permit the data set to be used for other analyses.

\section{Ethics approval and consent to participate}

The Oregon Health \& Science University's Institutional Review Board reviewed the study protocol, qualitative interview guides, and information sheet (interview data were not included in this manuscript).

\section{Consent for publication}

Not applicable.

\section{Competing interests}

Dr. McCarty served as an investigator on clinical trials using donated medications from Alkermes. All other authors declare that they have no competing interests.

\section{Author details}

${ }^{1}$ OHSU/PSU School of Public Health, Oregon Health \& Science University, 3181 SW Sam Jackson Park Road, Portland, OR 97239, USA. ${ }^{2}$ Center for Health Systems Effectiveness, Oregon Health \& Science University, 3181 SW Sam Jackson Park Road, Portland, OR 97239, USA. ${ }^{3}$ Oregon Health Authority, State Opioid Treatment Authority, 500 Summer Street, Salem, OR 97301, USA.
Received: 26 January 2019 Accepted: 8 August 2019

Published online: 15 August 2019

\section{References}

1. Olfson M, Wall M, Barry CL, Mauro C, Mojtabai R. Impact of Medicaid expansion on coverage and treatment of low-income adults with substance use disorders. Health Aff. 2018;37(8):1208-15.

2. Feder KA, Mojtabai R, Krawczyk N, Young AS, Kealhofer M, Tormohlen KN, et al. Trends in insurance coverage and treatment among persons with opioid use disorders following the Affordable Care Act. Drug Alcohol Depend. 2017;179:271-4.

3. Wen H, Hockenberry JM, Borders TF, Druss BG. impact of Medicaid expansion on Medicaid-covered utilization of buprenorphine for opioid use disorder treatment. Med Care. 2017;55(4):336-41.

4. Oregon Health Authority Public Health Division. Prescribing and Overdose Data for Oregon Salem, Oregon: Oregon Health Authority; 2019. https://www.oregon.gov/oha/ph/preventionwellness/substanceuse/ opioids/pages/data.aspx

5. Penfold RB, Zhang F. Use of interrupted time series analysis in evaluating health care quality improvements. Acad Pediatr. 2013;13(13):S38-44.

6. American Psychiatric Association. Diagnositic and Statistical Manual of Mental Disorders (DSM-5). 5th ed. Washington: American Psychiatric Association Publishing; 2013.

\section{Publisher's Note}

Springer Nature remains neutral with regard to jurisdictional claims in published maps and institutional affiliations.
Ready to submit your research? Choose BMC and benefit from:

- fast, convenient online submission

- thorough peer review by experienced researchers in your field

- rapid publication on acceptance

- support for research data, including large and complex data types

- gold Open Access which fosters wider collaboration and increased citations

- maximum visibility for your research: over 100M website views per year

At BMC, research is always in progress.

Learn more biomedcentral.com/submissions 